\title{
A Duality Theorem for Plastic Plates
}

\author{
By \\ W. H. Yang, Ann Arbor, Michigan \\ With 6 Figures \\ (Received February 20, 1987)
}

\begin{abstract}
Summary
Limit analysis studies the asymptotic behavior of elastic-plastic materials and structures. The asymptotic material properties exist for a class of ductile metals and are designed into optimal structural members such as I-beams and composite plates. The analysis automatically ignores the relatively small elastic deformations. Classical lower and upper bound theorems in the form of inequalities are mathematically incomplete. A duality theorem equates the greatest lower bound and the least upper bound. Although some general statement has been made on the duality relation of limit analysis. each vield criterion will lead to a specific duality theorem. The duality theorem for a class of plastic plates is established in this paper. The family of $\beta$-norms is used to represent the yield functions. Exact solutions for circular plates under a uniform load are obtained for clamped and simply supported boundaries as examples of the specific duality relations. Two classical solutions associated with Tresca and Johansen yield functions are also presented in the spirit of their own duality relations, providing interesting comparison to the new solutions. A class of approximate solutions by a finite element method is presented to show the rapid mesh convergence property of the dual formulation. Complete and general forms of the primal and dual limit analysis problems for the $\beta$-family plates are stated in terms of the components of the moment and curvature matrices.
\end{abstract}

\section{Introduction}

In the 1950s, limit analysis of plasticity [1], [2] had enjoyed a burst of development that produced many results of theoretical and practical significance. The advances made during that decade had laid the foundation of a potentially very powerful theory for plastic analysis and designs of structures [3] and metal forming processes [4]. Unfortunately, the development stopped short of a general algorithm for solving practical problems. It also left some important theoretical questions unanswered. As a result, limit analysis has not been in the main stream 
of solid mechanics compared to elasticity and incremental plasticity [5]. Some researchers abandoned limit analysis for its seemingly over simplified physical assumptions. Others were frustrated by its difficult mathematical structure. In fact, limit analysis involves deeper physical and mathematical concepts than one is led to believe. Its usefulness is irreplaceable by that of incremental plasticity. Limit analysis and incremental plasticity should complement each other to produce further advances in our endeavor of this branch of nonlinear mechanies.

Statements of "upper and lower bound theorems" in earlier articles of limit analysis were derived from simple physical arguments. Those inequality statements are mathematically incomplete because the equality relation between the least upper bound and the greatest lower bound had not been established. Such a relation, called duality, is essential for constructing a minimization or maximization algorithm that converges to the exact solutions of limit analysis problems.

Recently, duality theorems for limit analysis have received more attention in mathematical papers [6,7]. Although a general theorem [8] has been available. certain technicality in the pure mathematics language has not made it easy to apply the theorem to physically more spophisticated cases. Recent works like [9]. [15], [23] begin to close some gaps between mathematical, algorithmic and mechanical view points.

In this paper, we use a physically motivated upper bounding process to derive a class of duality theorems for plastic plates using a specific family of yield functions. Examples are presented with exact solutions approached from both lower and upper bounds. The theorems and examples should offer additional insight into the duality relations. Each specific yield function used in limit analysis will lead to an associated duality theorem.

The chosen $\beta$-family yield functions which span between the von Mises [10] and the Frobinius [15] functions provide an opportunity to examine the effects of yield functions on the limit solutions. Some solutions for circular plates in terms of static and kinematic quantities are obtained numerically. We present also the classical solutions using two other yield functions named after Tresca [12] and Johansen [13]. These two yield functions and the range of $\beta$-norms intersect. Therefore, the exact solutions associated with these two yield functions offer independent verification and comparison to the new solutions associated with the $\beta$-family yield functions.

In another section. a finite element method is used to obtain approximate solutions from the upper bound formulation or the dual. Because of the available exact solutions, the rapid convergence of the approximate solutions is numerically observed. One of the purposes of a duality theorem is to help constructing general algorithms for solving problems faced in engineering applications. Based on a duality theorem, a finite element method for general plate shapes, loadings and boundary conditions is given in [14] for the case $\beta=0$. 


\section{Family of Yield Functions Represented by $\beta$-Norms}

Since yield functions are convex and bounded, it is natural to represent a yield function by the notation of a mathematical norm (or semi-norm). A norm representation gives a direct perception of bounding certain quantities. For plastic plates, we shall bound the static quantity, the symmetric bending moment matrix [15],

$$
\mathbf{M}=\left(\begin{array}{ll}
M_{x x} & M_{x y} \\
M_{x y} & M_{y y}
\end{array}\right)
$$

such that its $\beta$-norm satisfies

$$
\|\mathbf{M}\|_{(\beta)}=\sqrt{M_{1}^{2}-\beta M_{1} M_{2}+M_{2}^{2}} \leqslant M_{0}
$$

where $M_{1}$ and $M_{2}$ are the eigenvalues of $\mathbf{M} ; \beta$ and $M_{0}$ are material constants. The square root function in (2) remains convex [16] for $-2 \leqslant \beta \leqslant 2$. We choose the range $0 \leqslant \beta \leqslant 1$ to fit some known yield functions. The case $\beta=1$ corresponds to the von Mises function and $\beta=0$ corresponds to the Frobinius norm of the matrix $\mathbf{M}$.

The yield loci, $\|\mathbf{M}\|_{(\beta)}=M_{0}$, form a family of ellipses in the $M_{1}-M_{2}$ plane as shown in Fig. 1 for the range of $\beta$ chosen. The Tresca yield criterion,

$$
\|\mathbf{M}\|_{T}=\max \left\{\left|M_{1}\right|,\left|M_{2}\right|,\left|M_{1}-M_{2}\right|\right\}=M_{0}
$$

and the Johansen yield criterion,

$$
\|\mathbf{M}\|_{J}=\max \left\{\left|M_{1}\right|,\left|M_{2}\right|\right\}=M_{0}
$$

intersect the range and their yield loci are shown in Fig. 1 by the hexagon and square respectively.

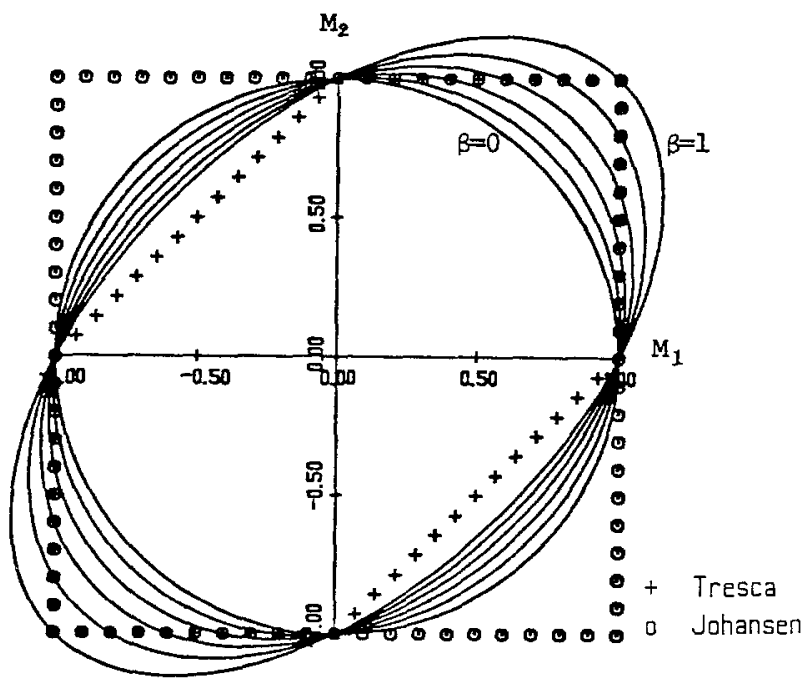

Fig. 1. $\beta$-family, Tresca and Johansen Yield Locı 
The $\beta$-norm family defined in (2) is introduced here for plastic plate analysis. It does not correspond to any standard mathematical norm except for $\beta=0$. We use a pair of parentheses on $\beta$ to avoid confusion from other known norms. The $\beta$-norm so defined satisfies all requirements of a mathematical norm [17].

\section{The Primal Formulation of Limit Analysis}

All solutions of mechanics problems satisfy three groups of equations (or relations) representing equilibrium, constitutive and kinematic conditions. We present a geometric interpretation of these solutions as point sets in some appropriate space.

Consider a system of algebraic equations whose number is less than the number of unknowns. The solution of the system if exists will not be unique. All solutions of such a system form a point set in the finite dimensional space of the unknown variables. If the system contains inequalities, its solution is naturally a point set.

Solutions of mechanics problems can also be represented by sets. Since differential and functional equations are usually involved in a mechanics problem, the solution set lies in a functional space. The study of differential equations and the spaces of their solutions is one of the most important activities in modern mathematics.

We shall now direct our discussion to plastic plate problems using this geometric concept. Let the solutions of equilibrium equation [15]

$$
\nabla \cdot(\nabla \cdot \mathbf{M})=q \varphi(x, y)
$$

and its static boundary conditions, if any, on the moment matrix $\mathbf{M}$, be represented by the statically admissible set $S$ in the space of $2 \times 2$ real symmetric matrix functions, $R^{2 \times 2}\left(R^{2}\right)$ and $\varphi(x, y)$ is a given normalized distribution function and $q$ is the non-negative multiplier of the distributed load. The limit analysis seeks the maximum $q$ such that the yield function.

$$
f(\mathbf{M})=\|\mathbf{M}\|_{(\beta)}-M_{0} \leqslant 0
$$

remains non-positive. Let the solutions of relation (6) be represented by the constitutively admissible set $C$ also in $R^{2 \times 2}\left(R^{2}\right)$. The primal (or natural) formulation of limit analysis becomes

$$
\begin{gathered}
\text { maximize } q(\mathbf{M}) \\
\text { subject to } \mathbf{M} \in L, L=S \cap C
\end{gathered}
$$

where $L$ is called the set of lower bound solutions. The set $L$ is non-empty since the trivial solution ( $\mathbf{M}=\mathbf{0}, q=0$ ) is always in $L$. Physically, there exist many elastic solutions corresponding to some small $q$ such that the inequality (6) remains strictly an inequality. Since the set $L$ is convex and bounded, there exists a unique maximum $q^{*}$ corresponding to one (or more) point in $L$. Any 
other point in $L$ corresponds to a value of $q<q$. This justifies the name "lower bound solutions" for points in $L$.

The lower bound formation (7) is in general difficult to solve because the finite dimensional approximation of a matrix function can be very large in dimension. The convex but generally nonlinear constraints in (7) present additional complexity in this maximization problem. For the circular plate problems considered, the exact solution of (7) can be approached by iteratively solving an ordinary differential equation. This will be demonstrated in a later section.

\section{Upper Bounding and Dual Formulation}

The equation of equilibrium (5) can be stated in an integral (or weak) form,

$$
\iint_{D} w \nabla \cdot(\nabla \cdot \mathbf{M}) d A=q \iint_{D} \varphi w d A
$$

where the integrals cover the plate domain $D$ and $w$ is an arbitrary function. Equation (8) is known as the virtual work statement in mechanics literature. Using the extension of divergence theorem for generalized functions [11] and natural boundary conditions . (8) can be rewritten in the form,

$$
q=\frac{\iint \mathbf{M}: \nabla \nabla w d A}{\iint \varphi w d A}
$$

where $\nabla \nabla w$ is the $2 \times 2$ Hessian (curvature) matrix of $w(x, y)$ and : denotes the inner product operator between two matrices [15]. Although second derivatives are involved in (9), we may not assume $w(x, y)$ to be twice differentiable in the classical sense. Engineers have long accepted solutions of plastic plate deformation with jump discontinuities in the first derivatives of $w$. Under a more relaxed condition of differentiability, the components of $\nabla \nabla w$ may contain distributions (integrable singularities). The function $w$ must also satisfy the kinematic boundary conditions if they are prescribed and the condition that the denominator of (9) does not vanish. The function $w(x, y)$, still arbitrary other than subjecting to these restrictions, belongs to the set $K \in R\left(R^{2}\right)$ which is called. kinematically admissible. Since $w$ appears homogeneously in both numerator and denominator of (9), we can scale $w$ to normalize the denominator such that

$$
\iint_{D} \varphi w d A=1
$$

which will be added to the conditions of kinematic admissibility. Since (9) is a statement of equilibrium. the task now is to maximize the integral in the numerator of (9) by choosing the optimal $\mathbf{M}$ in the constitutively admissible set $C$. 
Creating an upper bound of a quantity is a standard tool in mathematical analysis. But the sharpest upper bound to $q$ can only be established when none of the physical principles is violated. A constitutively admissible $\mathbf{M}$ not only satisfies the yield criterion (6), but is related also to the curvature matrix $\nabla \nabla w$ by the normality condition (flow rule) [18] such that

$$
\nabla \nabla w \propto \nabla f(\mathbf{M})
$$

We shall rewrite the relation (1I) in the principal directions for the specific $f(\mathbf{M})$ in $(6)$. Let $\kappa_{1}$ and $\kappa_{2}$ be the eigenvalues of $\nabla \nabla u$. Then

$$
\begin{aligned}
& k \kappa_{1}=\left(M_{1}-\frac{\beta}{2} M_{2}\right) /\|\mathbf{M}\|_{(\beta)} \\
& k \kappa_{2}=\left(M_{2}-\frac{\beta}{2} M_{1}\right) /\|\mathbf{M}\|_{(\beta)}
\end{aligned}
$$

where $k$ is a proportional factor. We can solve $M_{1}$ and $M_{2}$ in terms of $\kappa_{1}$ and $\kappa_{2}$ to obtain

$$
\begin{aligned}
& \frac{M_{1}}{\|\mathbf{M}\|_{(\beta)}}=\frac{k}{1-\beta^{2} / 4}\left(\kappa_{1}+\frac{\beta}{2} \kappa_{2}\right) \\
& \frac{M_{2}}{\|\mathbf{M}\|_{(\beta)}}=\frac{k}{1-\beta^{2} / 4}\left(\kappa_{2}+\frac{\beta}{2} \kappa_{1}\right)
\end{aligned}
$$

We now choose $\mu_{1}$ and $M_{2}$ most favorably to maximize $q$ without violating (6). The choices are those $\mathbf{M}$ on the yield loci under the condition,

$$
\|\mathbf{M}\|_{(\beta)}=M_{0}
$$

which is used to determine the proportional factor.

$$
k=\frac{\sqrt{1-\beta^{2} / 4}}{\sqrt{\kappa_{1}^{2}+\beta \kappa_{1} \kappa_{2}+\kappa_{2}^{2}}}
$$

Since the inner product of two matrices is invariant to coordinate rotation. we can choose $\mathbf{M}$ defined in (13) to establish an upper bound expression for the exact maximum $q^{*}$ such that

$$
q^{*} \leqslant \frac{M_{0}}{\sqrt{1-\beta^{2 / 4}}} \iint\|\nabla \nabla w\|_{(-\beta)} d A=\bar{q}(w)
$$

where the norm on $\nabla \nabla w$ with the sign of $\beta$ reversed is called the dual $\beta$-norm.

The dual formulation seeks the minimum value of $\bar{q}$ by choosing an optimal 
$w \in K$. The fact that

$$
\max q(\mathbf{M})=q^{*}=\min \bar{q}(w)
$$

is the duality condition sought. Since we know that $q(\mathbf{M}) \leqslant q^{*} \leqslant \bar{q}(w)$, we need to show only one equality case to establish (17). It will be shown in the next section. The dual formulation can be stated as

$$
\text { minimize } \bar{q}(w)
$$

$$
\text { subject to } w \in K
$$

We may regard (7) and (18) to be dual of each other depending on me is view point. In fact, some mathematical work on the subject starts with a lik. of (18) as the natural formulation and derives its dual in a like of (7).

\section{Circular Plates Under Uniform Load}

We shall now demonstrate the duality relation (17) by the examples of a circular plate of radius $\mathrm{R}$ under uniform load $q$. Solutions are obtained by maximizing $q$ and minimizing $\bar{q}$ for the range of $\beta$. using two types of boundary conditions: simply supported and clamped. By axisymmetry, the moment and curvature matrices are both diagonal such that

$$
\mathbf{M}=\left(\begin{array}{cc}
M_{r}(r) & 0 \\
0 & M_{\theta}(r)
\end{array}\right) \quad \nabla \nabla w=\left(\begin{array}{cc}
\frac{\mathrm{d}^{2} w}{d r^{2}} & 0 \\
0 & \frac{1}{r} \frac{d w}{d r}
\end{array}\right)
$$

(A) Greatest Lower Bound: The equilibrium equation for the circular plates can be integrated once from (5) or directly formulated from an axisymmetric plate element to give

$$
\frac{d M_{r}}{d r}+\frac{1}{r}\left(M_{r}-M_{0}\right)=\frac{q r}{2}
$$

which is a first order ordinary differential equation in two unknowns $M_{\imath}(r)$ and $M_{\theta}(r) . M_{\theta}$ can be solved in terms of $M$, by the choices of $\mathbf{M}$ on the yield locus such that

$$
M_{\theta}=\frac{\beta}{2} M_{r}-\sqrt{M_{\theta}^{2}-\left(1-\beta^{2} / 4\right) M_{r}^{2}}
$$

where the negative sign of the square root is chosen since $M_{\theta} \leqslant M_{r}$ for $q \geqslant 0$. The intersection of $S$ as the solution set of (20) and $C$ as the solution set of (21) can be obtained by substituting (21) into (20). The resulting differential equation in $M_{r}(r)$ can be integrated numerically with the "initial" condition

$$
\frac{M_{r}(0)}{M_{0}}=-\frac{1}{\sqrt{2-\beta}}
$$




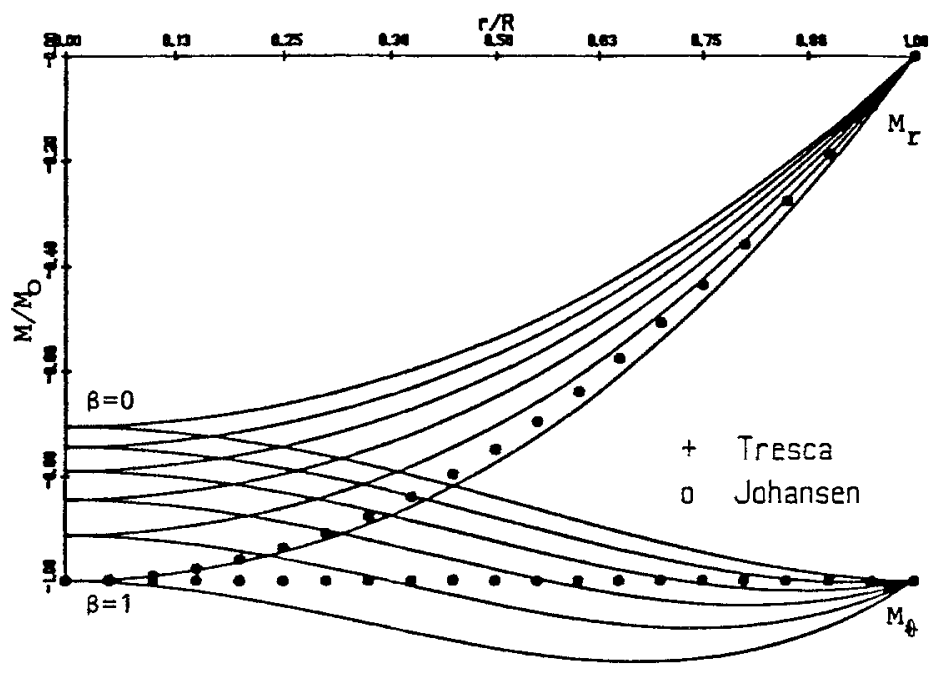

Fig 2. Moment distribution in a simply supported circular plate

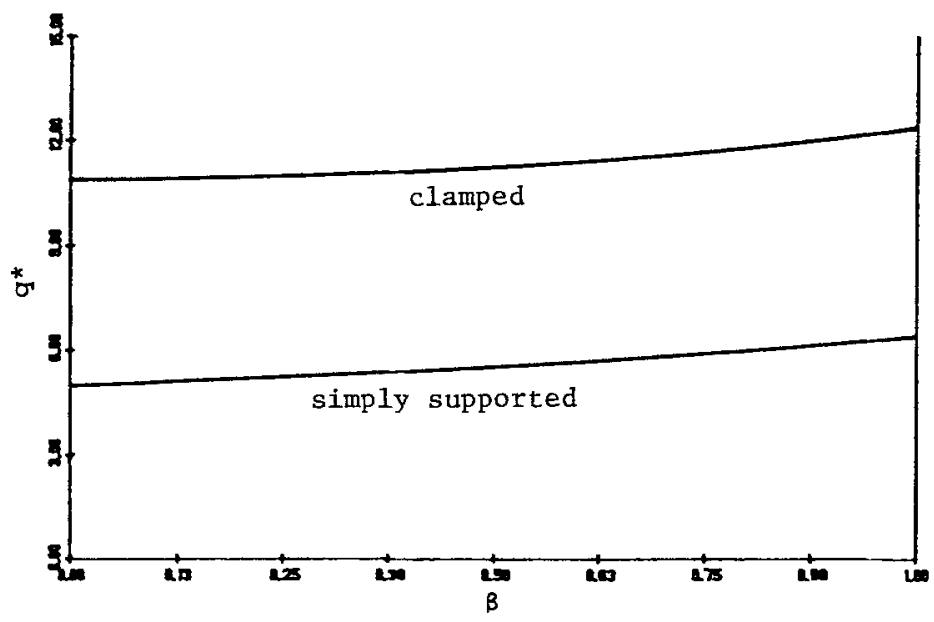

Fig. 3. Collapse loads of $\beta$-family plates

An appropriate value of $q$ is yet to be chosen to satisfy also the "end" condition

$$
\frac{M_{r}(R)}{M_{0}}= \begin{cases}0 & \text { simply supported } \\ 2 / \sqrt{4-\beta^{2}} & \text { clamped }\end{cases}
$$

where $2 / \sqrt{4-\beta^{2}}$ is the maximum attainable value of $M_{r}$.

With a stable numerical method, the integration can be made as accurately as one desires. We choose the step size $\mathrm{h} / R=0.01$ and use a modified Newton's iteration to home-in on the correct value of $q$ which is the greatest lower bound or the collapse load $q^{*}$. 


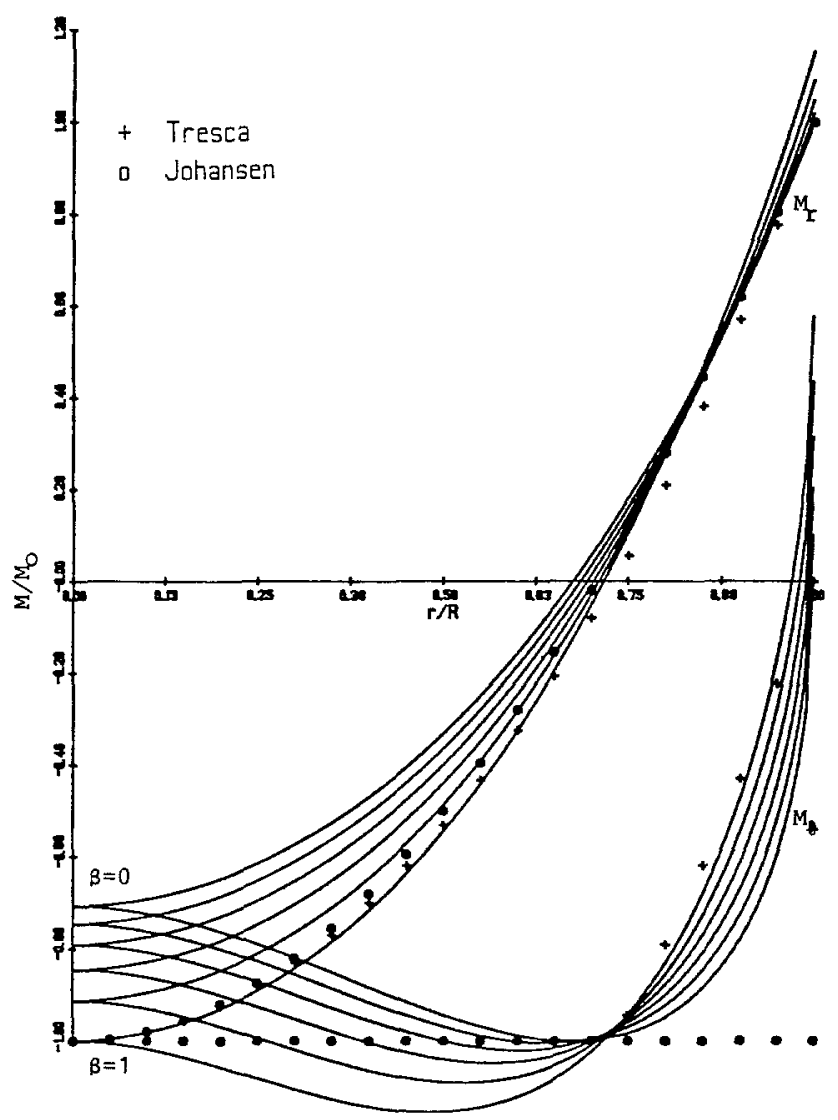

Fig. 4. Moment distribution in a clamped circular plate

The results of the moment distribution $M_{r}(r), M_{\theta}(r)$ are shown in Figs, 2 and 4 for simply supported and clamped plates respectively. The collapse loads $q^{*}$ plotted as functions of $\beta$ for both cases of boundary conditions are shown in Fig. 3. These results can be regarded as exact since further reduction of step size of integration will not change the results in six significant digits in our single precision computation.

The collapse load increases as $\beta$ increases. Although this result is expected, it is not obvious since the constitutively admissible set $C$ corresponding to a given $\beta$ is not a subset of that of a larger $\beta$. It is conceivable that for certain plate deformations whose moments lie in the second and fourth quadrants of Fig. 1, larger $\beta$ may produce smaller collapse load.

The static solutions presented in Figs. 2, 3 and 4 tell only one-half of the limit analysis story. If we need to know the collapse modes, the dual problem (18) must be solved. We shall next discuss the kinematic solutions of the circular plates considered above. 
(B) Least U'pper Bound: L'sing the variational calculus, we can derive the "Euler"s equation" for the minimizing functional of (18). After some manipulation, we obtain the following ordinary differential equation

$$
\frac{d}{d r}(r p)-\frac{\beta}{2} p-\sqrt{1-\beta^{2} / 4} \sqrt{1-p^{2}}=B r^{2}
$$

where $p=\frac{r w^{\prime \prime}+\beta w^{\prime} / 2}{\sqrt{\left(u u^{\prime \prime}\right)^{2}+\beta r w^{\prime} w^{\prime \prime}+\left(w^{\prime}\right)^{2}}}$ with $w^{\prime \prime}=\frac{d^{2} u}{d r^{2}}: u^{\prime}=\frac{d u}{d r}$ and $B$ is an arbitrary constant of integration. Since $w^{\prime \prime}=w^{\prime} / r$ at $\mathrm{r}=0$, we obtain the initial condition,

$$
p(0)=\frac{\sqrt{2+\beta}}{2}
$$

With a trial value of $B \cdot p(r)$ can be numerically integrated.

Let $\theta=d w / d r$. It is easy to show that $\theta$ satisfies the differential equation

$$
r \frac{d \theta}{d r}=\left(p \sqrt{\frac{1-\beta^{2} / 4}{1-p^{2}}}-\frac{\beta}{2}\right) \theta
$$

and $\theta(0)=0$. Equation $(26)$ can be readily integrated with a known $p(r)$. Finally, the differential equation $d w / d r=\theta(r)$ and $w(R)=0$ provides the solution $w(r)$. Thus far, the trial solutions $p(r), \theta(r)$ and $w(r)$ depend on the assumed value of $B$. We can use these trial solutions to evaluate $\bar{q}(B)$. The correct $B$ associated with $\bar{q}_{\mathrm{m} n}$ can be determined iteratively using the condition $d \bar{q} / d B=0$. The algorithm is summarized below:

1. Assume a value of $B$

2. Integrate $p(r)$

3. Integrate $\theta(r)$

4. Integrate $w(r)$

5. Calculate $\bar{q}(B)$

6. Minimize $\bar{q}(B)$ iteratively by repeating from step 1 with a better choice of $B$.

Note that we have only imposed $w(R)=0$ as the kinematical boundary condition. For the clamped plate, it may be surprising to some that $\theta(R)$ may not ranish as it does in the linear plate theories. The class of kinematic solutions in limit analysis of plates admits functions with discontinuous first derivative. This leads to the relaxed boundary condition to allow nonzero slope as the plate approaches a clamped boundary. The slope then takes a jump to the zero value imposed. Interpretation of $u^{\prime \prime}$ at a clamped boundary is a distribution (a line singularity) which makes a contribution in the integration of $\bar{q}$. This phenomenon known as a yield line can also take place in the interior of plates [13].

We apply standard library subroutines to integrate the ODEs and to minimize a real function. The kinematic solutions $w(r)$ are shown in Figs. 5 and 6 for simply supported and clamped plates respectively. The minimum values of $\bar{q}$ 


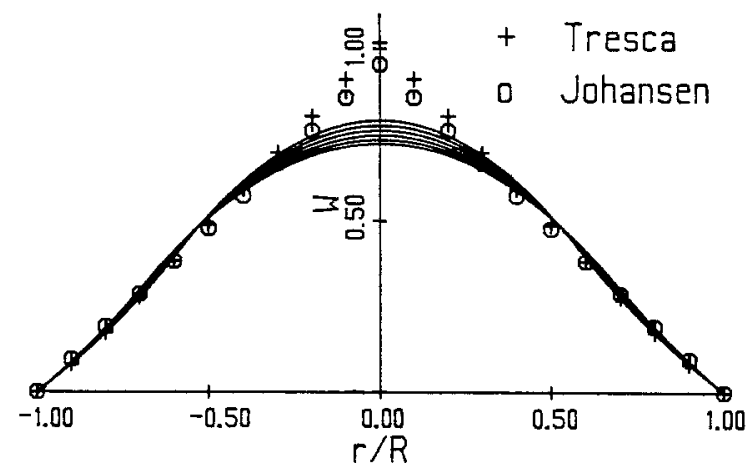

Fig. 5. Collapse modes of simply supported circular plates

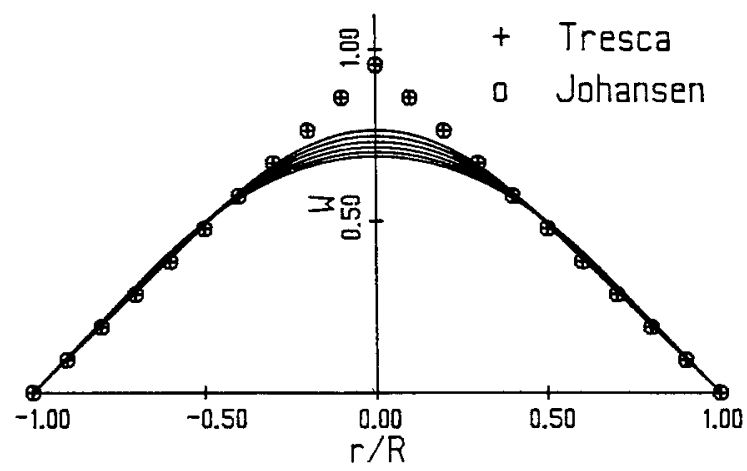

Fig. 6. Collapse modes of clamped circular plates

agree with that of $q^{*}$ shown in Fig. 3 to five significant digits for all values of $\beta$ with our single precision computation. We conclude that these minimum solutions are practically exact. We have demonstrated the duality relation (17) by the solutions of $\beta$-family circular plates. In addition, we present in the next section two classical solutions which satisfy their own duality relations.

\section{Classical Solutions}

The piecewise linear yield functions of Tresca and Johansen seem popular in earlier works of plasticity. Although, Tresca function in stress space has been tested experimentally [19], its usage as a function of moment to model plastic plate behavior was only conjectured. It was chosen for its simplicity rather than experimental fitness. For circular plates, the greatest lower bound solutions for these two yield criteria are given in [20]. The least upper bound solutions are presented in [21], [22]. 
For a simply supported circular plate under uniform load. the kinematic solutions for both yield criteria are the cone

$$
w(r)=\frac{3}{\pi R^{2}}\left(1-\frac{r}{R}\right)
$$

where $w$ has dimension of $R^{-2}$ because of the normalization condition (10). The cone solution remains the same for clamped Johansen plate. For the clamped Tresca plate, a cone of the inner region is matched to a logarithmic function for the outer region to give

$$
w(r)= \begin{cases}A[1-r / a+\ln (R / a)] & 0 \leqslant r \leqslant a \\ \mathrm{~A} \ln (R / r) & a \leqslant r \leqslant R\end{cases}
$$

where $A=6 /\left[\pi\left(3 R^{2}-a^{2}\right)\right]$ and $a=0.730012 R$. The collapse loads are: $q^{*}=6 M_{0} /$ $R^{2}$ for both simply supported Tresca and Johansen plates; $q^{*}=12 M_{0} / R^{2}$ for the clamped Johansen plate and $11.258777 M_{0} / R^{2}$ for clamped Tresca plate.

The static solutions for the simply supported Tresca and Johansen plates in terms of moment components are the same

$$
M_{r}(r)=M_{0}\left(r^{2} / R^{2}-1\right), \quad M_{0}(r)=-M_{0}
$$

For the clamped Johansen plate, they are simply

$$
M_{r}(r)=M_{0}\left(2 r^{2} / R^{2}-1\right) . \quad M_{\theta}(r)=-M I_{0}
$$

The clamped Tresca plate has the two-piece matehed solution,

$$
\begin{aligned}
& M_{\imath}(r)= \begin{cases}M_{0}\left[(r / a)^{2}-1\right] & 0 \leqslant r \leqslant a \\
M_{0}\left[1.5(r / a)^{2}-1.5-\ln (r / a)\right] & a \leqslant r \leqslant R\end{cases} \\
& M_{\theta}(r)= \begin{cases}-M_{0} & 0 \leqslant r \leqslant a \\
M_{0}\left[1.5(r / a)^{2}-2.5-\ln (r / a)\right] & a \leqslant r \leqslant R\end{cases}
\end{aligned}
$$

where $a=0.730012 R$ is the point of matching.

These exact static and kinematic solutions are shown as the dotted lines in Figs. 2. 4, 5 and 6. We shall draw some conclusions from the comparison of these classical solutions and the new solutions obtained in the last two sections.

\section{Approximate Upper Bound Solutions}

Since the dual formulation involves only a real unknown function $u$, it is in general easier to approximate its solution by a finite difference or a finite element method. A general finite element method and some solutions of circular and square plates are given in [14]. We shall present here solutions of a circular plate obtained by a one dimensional finite element method where $w(r)$ is approximated by piecewise cubic spline functions with continuity of $w$ and $\theta$ at element boundaries. 
Table 1. Upper bound solutions q approximated by two cubic spline elements compared to the exact solutions $q^{*}$

\begin{tabular}{cccc}
\hline$\beta$ & $\bar{q}$ & $q^{*}$ & \%Error \\
\hline 0.0 & 5.214663 & 4.998523 & 4.32 \\
0.1 & 5.295504 & 5.090353 & 4.03 \\
0.2 & 5.385060 & 5.189316 & 3.77 \\
0.3 & 5.484692 & 5.296411 & 3.55 \\
0.4 & 5.596005 & 5.412847 & 3.38 \\
0.5 & 5.720942 & 5.540108 & 3.26 \\
0.6 & 5.861901 & 5.680030 & 3.20 \\
0.7 & 6.021901 & 5.834934 & 3.20 \\
0.8 & 6.204817 & 6.007777 & 3.38 \\
0.9 & 6.415734 & 6.202425 & 3.44 \\
1.0 & 6.661482 & 6.424049 & 3.70 \\
\hline
\end{tabular}

Cising only two elements in the domain, $0 \leqslant r \leqslant R$, we obtain the following results for the simply supported plate and compare them with the exact solutions.

When eight elements are used, the results agree with the exact solutions to all six decimals. Such rapid convergence in the mesh size is highly desirable for this nonlinear problem since the finite dimensional problem is solved repeatedly for iterations and parameter variations on $\beta$. Decreasing the dimension of the problem dramatically improves computational efficiency.

\section{Conclusion}

The trend of plastic analysis today is heavily inclined toward the large scale incremental computation. Two major drawbacks remain in this approach. High computational cost is one. Lack of exact solutions to make test cases for highly complex codes often raises questions on the quality of the numerical solutions they produce. After a tremendous computing effort and cost, a researcher may only conclude that the results seem reasonable compared with some experiments. Incremental computation produces a great deal of details not required in the intended analysis but necessary for the subsequent incremental computation and adding up costs. Of course, some applications like residual stress analysis can only be made by incremental computation.

Limit analysis computations are relatively less expensive, therefore more useful in designs as well as parametric analysis of material or process modelling where computations are repeated for variations and iterations. More exact solutions are available in limit analysis to check general computer codes than they are in incremental plasticity. Limit analysis bypasses the solutions of intermediate elastic-plastic deformation and computes directly the more interesting limit solutions. Limit solutions are often the only information sought in design criteria. At the present, the activities of incremental computations seem to 
overwhelm other efforts in plasticity research. Limit analysis and incremental analysis are equally important and should complement each other to further advance the plasticity theory.

The exact limit solutions for the $\beta$-family vield functions should be a welcoming addition to the limited repertoire of exact solutions in plasticity. The $\beta$-family can fit a wide range of experimental data on vield loci. The exact solutions in this paper may be used in conjunction with circular plate experiments to identify a specific material model.

The results of the renowned experiment of Taylor and Quinney [19] faror the von Mises yield function to that of Tresca which claims validity in single crystal experiments. Since the random orientations of micro structures in materials, the polycrystal aggregates like most metals hehave as a statistical mean of their single crystal properties. A smooth yield function models the macroscopic behavior more realistically than a piecewise yield function. Lpon examining the limit solutions of the $\beta$-family plates and that of Tresca and Johansen plates. one should notice the consistancy of their intersecting relations. However, the moment distributions and the deflection modes of the $\beta$-plates are more consistant to that observed in real plates. The sharp apex of a cone associated with kinematic solutions of Tresca and Johansen plates has never been observed in experiments of a plate under uniform load.

A smooth yield function has its computational advantage. The $\beta$-family has vielded smooth solutions in the interior of the plate. Absence of yield lines in the interior greatly simplifies the finite element approximation. There is no need to be concerned with integrating the possible distributions not known a priori in a plate. The general finite element method in [14] produced smooth solutions even for square plates. Non-smoothness in loading and boundary conditions may still introduce vield lines and apices in the interior. We are currently investigating this problem.

Finally, we reiterate a point made earlier. An upper or lower bound inequality does not constitute a useful limit analysis theorem. Only a duality theorem which equates the least upper bound to the greatest lower bound becomes the basis for constructing maximization and minimization algorithms for limit solutions. Since they are stated in matrix notation, the duality theorem (17) and the primal and dual problems (7) and (18) apply as well to general plate shapes as to those circular plates presented in this paper. We shall restate these two problems in $a(x, y)$ coordinate system in terms of the moment and curvature components.

\section{(A) The Primal Problem:}

maximize $q(\mathbf{M})$

subject to:

$$
\frac{\partial^{2} M_{x x}}{\partial x^{2}}+2 \frac{\partial^{2} M_{x y}}{\partial x \partial y}+\frac{\partial^{2} \boldsymbol{M}_{y y}}{\partial y^{2}}=q \varphi(x, y)
$$




$$
\sqrt{\frac{2-\beta}{4}\left(M_{x x}+M_{y y}\right)^{2}+\frac{2+\beta}{4}\left[\left(M_{x x}-M_{y y}\right)^{2}+4\left(M_{x y}\right)^{2}\right]} \leqslant M_{0}
$$

static boundary conditions.

(B) The Dual Problem:

$$
\text { minimize } \bar{q}(w)
$$

subject to:

$$
\begin{aligned}
& \bar{q}=\frac{M_{0}}{\sqrt{1-\beta^{2} / 4}} \cdot \\
& \iint \sqrt{\frac{2+\beta}{4}\left(\frac{\partial^{2} w}{\partial x^{2}}+\frac{\partial^{2} w}{\partial y^{2}}\right)^{2}+\frac{2-\beta}{4}\left[\left(\frac{\partial^{2} w}{\partial x^{2}}-\frac{\partial^{2} w^{2}}{\partial y^{2}}\right)^{2}+4\left(\frac{\partial^{2} w}{\partial x \partial y}\right)^{2}\right]} d A \\
& \iint w(x, y) \varphi(x, y) d A=1
\end{aligned}
$$

kinematic boundary conditions,

which are convenient for constructing numerical algorithms for general plate shapes, loadings and boundary conditions. A numerical method for the dual problem should allow possible jump discontinuity in the first derivatives of $u(x, y)$ and provide accurate integration of the contribution along the lines of discontinuity. These deeper issues of numerical analysis will be discussed in a separate paper.

\section{References}

[1] Hill, R.: The mathematical theory of plasticity. Oxford Clarendon Press 1950.

[2] Prager. W., Hodge. P. G., Jr : Theory of perfectly plastic solids. John Wiley \& Sons 1951.

[3] Hodge. P G., Jr. Plastic analysis of structures. McGraw-Hill 1959.

[4] Sokolorski, V. V.: The construction of stress and velocity fields in plastic flow problems. Inzhenernyi Zh. 1 (3). 116-121 (1961).

[5] Balmer, H. A.. Doltsinis. J. St.. Konig. M.: Elasto-plastre and creep analysis with the ASKA program system. Comp. Meths. Appl. Mech. Eng. 3, 87-104 (1974).

[6] Strang, G.: A mimimax problem in plasticity. In F Functional analysis and numerical analysis (Nashed, M., ed.), pp. 319-333. Springer Lecture Notes 70I (1979).

[7] Temam, R., Strang, G.. Duality and relaxation in the variational problems of plasticity. J. Mecanique 19 (3), 493-52l (1980).

[8] Lions, J. L., Stampacchia, G.: Variational inequalities. Comm. Pure Appl. Math. 20, 493-519 (1967).

[9] Christiansen, E., Larsen, S.. Computations in limit analysis for plastic plates. International J. Num. Meth. Eng., 19, 169-184 (1983)

[10] ron Mises, R.: Göttinger Nachrıchten. Math. Phys. Klasse (1913). 
[11] Fleming, W. H. Functions with generalized gradient and generalized surfaces. Ann. Mat. Pure Appl., Series 4, 44, 93-103 (1957).

[12] Tresca, H. Comptes Rendus Acad Sci. Paris 59 (1864).

[13] Johansen. K. W.: Yield line theory. Original published by the Danish Cement and Concrete Association, 1943, translated and published by William Clowes and Sons. London, 1962.

[14] Yang. W. H., Young. S. L.: A finite element approach to limit solutions of plates. FENOMECH84, Stuttgart. Sept. 10-14. 1984.

[15] Yang, W H.: A variational principle and an algorithm for limit analyssis of beams and plates. Comp. Meth. Appl. Mech. Eng. 33, 575-582 (1982).

[16] Yang, W. H. A generalized von Mises criterion for yield and fracture. J. Appl. Mech. 47, 297-300 (1980).

[17] Isaacson, E., Keller, H.: Analysis of numerical methods. John Wiley \& Sons 1966.

[18] Drucker, D. C.: A more fundamental approach to plastic stress-strain relations. 1st U.S. Congr. Appl. Mech., pp. 487-491 (1952).

[19] Taylor, G. I., Quinney, H.. Phil. Trans Roy. Sor. A 230 (1931)

[20] Hopkins, H G., Prager, W.: The loading carrying capacity of circular plates. J Mech. Phys. Solids 2, 1-13 (1953)

[21] Yang, W H. Minimization approach to limit solutions of plates. Comp. Meth. Appl. Mech. Eng. 28, 265-274 (1981).

[22] Mansfield, E. H.: Studies in collapse analysis of rigid-plastic plates with a square yreld diagram. Proc. Roy. Soc. London, Ser. A 241, 311-338 (1957).

[23] Overton, M. L.: A quadratically convergent method for minimizing a sum of Euclidean norms Mathematical Programming 27, 34-63 (1983).

\author{
W. H. Yang \\ The University of Michigan \\ College of Engineering \\ Department of Mechanical Engineering and \\ Applied Nechanies \\ 321 W. E. Lay Automotive Lab, N.C. \\ Ann Arbor, MI 48109-2121 \\ U.S.A.
}




\title{
Forced Vibrations of an Elasto-Plastic and Deteriorating Beam*
}

\author{
By \\ P. Fotiu, H. Irschik and F. Ziegler, Wien, Austria
}

With 8 Figures

(Received February 27, 1987)

\begin{abstract}
Summary
A solution method for elastoplastic vibrating beams including damage accumulation is shown, where inelastic behavior of the structure is represented by an additional loading due to sources of selfstresses acting upon the linear elastic structure of time-invariant stiffness. Response due to this additional loading is evaluated using proper Green's functions. Thus, integral relations are set up, similar to Maysel's formula. Theory is applied to a two span sand wich beam with elastoplastic degrading flanges and elastic core material
\end{abstract}

\section{Introduction}

Dynamic plastic deformations in severely loaded structures render damping and the rather unwanted effects of drift (permanent strain) and damage accumulation during cycling. Quasistatic analysis is effectively performed by the standard incremental stiffness formulation often in connection with a diseretization by the Finite-Element-Method. Extension to the dynamic problem runs high in computing time. An initial strain formulation summarized by Lin [1] was not very well received until recently an extension to dynamic plasticity showed the merits of the method [2]. A comparative study was made by Argyris et al. [3].

Improvements were performed when Irschik and Ziegler [4], [5] consequently introduced the concept of sources of selfstresses (SES) determined by the nonlinear (plastic) part of total strain and acting upon the associated linear elastic system when keeping the stiffness constant in time. Recognizing the full analogy to thermal shock loading of the linear elastic beam, the powerful superposition

* Part of the lecture on "Nonstationary Vibrations of Yielding Structures" contributed by F. Ziegler in the Session on Computational Plasticity (Organizers. S. N. Atluri and D. W. Nicholson) to the Aris Phillips memorial symposium (chairman: M Eisenberg. Gainesville, Florida Jan. 28-30, 1987). 